\title{
ON THE EXISTENCE OF INCOMPRESSIBLE SURFACES IN CERTAIN 3-MANIFOLDS
}

\author{
WOLFGANG HEIL
}

If $M$ is the closure of the complement of a regular neighborhood of a nontrivial knot in $S^{3}$ then there exists a nonsingular torus $T$ embedded in $M$, which is incompressible (i.e. the inclusion $i: T \rightarrow M$ induces a monomorphism $\left.i_{*}: \pi_{1}(T) \rightarrow \pi_{1}(M)\right)$. If $F$ is any orientable closed incompressible surface embedded in $M$ then $\pi_{1}(M)$ contains $\pi_{1}(F)$ as a subgroup. L. Neuwirth [3, Question T] asks whether the converse is true: If $\pi_{1}(M)$ contains the group $\mathcal{F}$ of a closed (orientable) surface of genus $g>1$, does there exist a nonsingular closed surface $F$ of genus $g$ whose fundamental group is injected monomorphically into $\pi_{1}(M)$ by inclusion? As a partial answer we show that not for every such $\mathcal{F} \subset \pi_{1}(M)$ there exists an incompressible $F \subset M$. The question remains open whether $M$ contains incompressible closed surfaces of genus $>1$. We show that for torus knots $M$ does not contain such surfaces, by showing that $\pi_{1}(M)$ does not contain subgroups $\mathfrak{F}$.

1. Isotopic surfaces. Let $M$ be a compact 3-manifold (orientable or nonorientable). A "surface $F$ in $M$ " always means a 2 -sided embedded surface $F$ in $M$ such that $F \cap \partial M=\partial F . F$ is incompressible in $M$ iff $F \neq S^{2}$ and $\operatorname{ker}\left(i_{*}: \pi_{1}(F) \rightarrow \pi_{1}(M)\right)=1$, where $i: F \rightarrow M$ is the inclusion. We say $M$ is $P^{2}$-irreducible iff $M$ is irreducible (every 2 -sphere bounds a ball) and does not contain (2-sided) projective planes. $M$ is called boundary-irreducible iff $\partial M$ is a system of incompressible surfaces.

Theorem 1. Let $M$ be a $P^{2}$-irredubicle 3-manifold. Let $G$ be an incompressible surface in $M$ and $\mathfrak{F} \subset i_{*} \pi_{1}(G) \subset \pi_{1}(M)$. If there exists an incompressible surface $F \subset M$ such that $\partial F \subset \partial G \cap \partial F$ and $i_{*} \pi_{1}(F)=\mathfrak{F}$, then $F$ is isotopic to $G$.

This follows from theorems obtained by Waldhausen [5]. In particular we need the following:

Proposition [5, Proposition 5.4]. Let $M$ be $P^{2}$-irreducible. Let $F$ and $G$ be incompressible surfaces in $M, \partial F \subset \partial F \cap \partial G$, such that $F \cap G$ consists of mutually disjoint simple closed curves (with transversal intersection at any curve which is not in $\partial F$ ). Let $H$ be a surface and suppose there is a map $f: H \times I \rightarrow M$ such that $f \mid H \times 0$ is a covering map onto $F$

Received by the editors April 21, 1969. 
and $f(\partial(H \times I)-H \times 0) \subset G$. Then there is a surface $\widetilde{H}$ and an embedding $\tilde{H} \times I \rightarrow M$ such that $\tilde{H} \times 0=\tilde{F} \subset F ; \operatorname{Cl}(\partial(\widetilde{H} \times I)-\tilde{H} \times 0)=\tilde{G} \subset G$ and $\tilde{F} \cap G=\partial \tilde{F}$; moreover if $\tilde{G} \cap F \neq \partial \tilde{G}$ then $\tilde{F}$ and $\tilde{G}$ are discs.

Waldhausen proves this for orientable $M, F, G$, using his Lemmas 5.1 to 5.3 in [5]. In the nonorientable case 5.1 of [5] may be proved by looking at the orientable 2 -sheeted covering of $M$ (see [2]). Then the proofs of Lemmas 5.2 to 5.4 in [5] go through in the nonorientable case as well, noting that $F$ and $G$ are 2-sided in $M$.

Proof of the theorem. Suppose $F$ exists. By small isotopic deformations, constant on $\partial M$, we may assume that $G \cap F$ consists of a system of closed curves, the number of which is minimal. We claim: There exists a surface $H$ homeomorphic to $F$ and a map $f: H \times I \rightarrow M$ such that $f \mid H \times 0$ is a homeomorphism onto $F$ and $f \mid(H \times 1 \cup \partial H \times I)$ $C G$. For, let $f \mid H \times 0$ be $i: F \rightarrow M$. Since $\mathfrak{F} \subset \pi_{1}(G)$ and $\partial F \subset \partial F \cap \partial G$, we can define the map on $H \times 0 \cup \partial H \times I \cup H^{(1)} \times I$, where $H^{(1)}$ is the 1-skeleton of $H$, such that $f \mid H^{(1)} \times 1 \subset G$. Since $G$ is incompressible, we can extend this map to a map from $\partial(H \times I) \rightarrow M$. Now $\pi_{2}(M)=0$ (by our assumption on $M$ and the projective plane theorem [1]; in fact it follows from the Hurewicz-isomorphism on the universal cover that $M$ is aspherical), therefore $f$ can be extended to a map $H \times I \rightarrow M$. The rest of the proof copies the proof of Corollary (5.5) in [5]: by the proposition, there exist pieces $\tilde{G} \subset G$ and $\tilde{F} \subset F$ which are parallel in $M$ such that $\tilde{F} \cap G=\partial \tilde{F}$. If $\tilde{G} \cap F \neq \partial \widetilde{G}$ then $\tilde{F} \cup \tilde{G}$ bounds a ball, since $M$ is irreducible. This ball contains a piece $F^{\prime} \subset F$. Deforming $F^{\prime}$ out of this ball across $\tilde{G}$, we could make $F \cap G$ smaller, a contradiction. Hence we have $\tilde{G} \cap F=\partial \widetilde{G}$. Therefore there exists an isotopic deformation of $F$ (constant on $F-\widetilde{F}$ ) which throws $\widetilde{F}$ onto $\widetilde{G}$. If $\widetilde{F}$ would not be all of $F$, then we could deform $\widetilde{F}-\partial F \cap \widetilde{F}$ out of $G$ (keeping $\partial F$ fixed) and thereby reduce the intersection number $F \cap G$. Hence $F=\widetilde{F}, F \cap G=\partial F \subset \partial F \cap \partial G$, hence $\partial \widetilde{G}=\widetilde{G} \cap F \subset \partial M$ and since $G \cap \partial M=\partial G$ we have $G=\widetilde{G}$.

Let $\mathfrak{F}$ be a subgroup of $\pi_{1}(M)$. We say $\mathfrak{F}$ is carried by a surface $F \subset M$ iff there exists an embedding $i: F \rightarrow M$ such that $i_{*} \pi_{1}(F)=\mathfrak{F}$ and ker $i_{*}=1$.

Corollary. Let $M$ be $P^{2}$-irreducible. Let $G$ be a closed incompressible surface of genus $>1$ in $M$. Then there exists a subgroup $\mathfrak{F} \subset \pi_{1}(M)$ which is not carried by a surface $F \subset M$ but is isomorphic to $\pi_{1}(F)$. (In fact, if $G$ is not a Klein bottle there exist infinitely many nonisomorphic subgroups of $\pi_{1}(M)$ having this property.)

Proof. Let $F$ be a finite covering of $G$ such that $F$ is not homeomorphic to $G$. (Since $G \neq S^{2}, P^{2}$, Torus, Klein bottle, we can construct 
infinitely many topologically different compact $F$ 's.) Then $p_{*} \pi_{1}(F)=\mathfrak{F}$ (where $p: F \rightarrow G$ is the covering map) is a subgroup of $\pi_{1}(G)$, hence of $i_{*} \pi_{1}(G) \subset \pi_{1}(M)$. If $\mathfrak{F}$ would be carried by $F$, then by Theorem $1, F$ would be isotopic to $G$, a contradiction.

In particular this corollary applies to complements of nontrivial knots as mentioned in the introduction.

2. Surfaces in 3-manifolds which groups have a center. Let $\mathfrak{F}$ be the fundamental group of a closed surface $F$. If $F$ is orientable suppose genus $(F)>1$, if $F$ is nonorientable let genus $(F)>2$.

Lemma. Let $M$ be an irreducible (compact) 3-manifold with $\pi_{1}(M)$ $\approx \mathfrak{F} \times \mathrm{Z}$, then $M$ is a fibre bundle over $S^{1}$ with fiber $F$.

This is a special case of Stallings theorem [4].

Theorem 2. Let $M$ be a $P^{2}$-irreducible, boundary irreducible 3manifold and suppose the center 3 of $\pi_{1}(M)$ is infinite. If $\partial M \neq \varnothing$, then $\pi_{1}(M)$ does not contain a subgroup $\mathfrak{F}$ as above.

Proof. Suppose there exists $\mathfrak{F} \subset \pi_{1}(M)$. Then, since the center of $\mathfrak{F}$

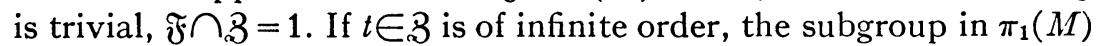
which is generated by $\mathfrak{F}$ and $t$ is isomorphic to $\mathfrak{F} \times \mathbf{Z}(t)$. If $D(M)$ denotes the double of $M$, then since $M$ is boundary irreducible, $i_{*}$ : $\pi_{1}(M) \rightarrow \pi_{1}(D(M))$ is a monomorphism, where $i: M \rightarrow D(M)$ is the inclusion (this is well known; a proof may be found, e.g., in [4]). Since $D(M)$ is $P^{2}$-irreducible and $\pi_{1}(D(M))$ not finite, $D(M)$ is aspherical (see the remark in the proof of Theorem 1). Therefore we can construct a map $f: F \times S^{1} \rightarrow D(M)$ which induces the embedding $\mathfrak{F} \times \boldsymbol{Z} \rightarrow \pi_{1}(M) \stackrel{i_{*}}{\rightarrow} \pi_{1}(D(M))$. It follows from Waldhausen's theorem [5, Theorem 6.1] (see [2] for the nonorientable case), that $f$ is homotopic to a covering map. In particular, since $F \times S^{1}$ is compact it follows that $\mathfrak{F} \times \boldsymbol{Z}$ has finite index in $\pi_{1}(D(M))$ and therefore in $\pi_{1}(M) \subset \pi_{1}(D(M))$. Now consider the covering $\tilde{M}$ of $M$ which is associated to $\mathfrak{F} \times \boldsymbol{Z}$. $\tilde{M}$ is compact. Now the universal covering of $M$ can be embedded in a ball such that the interior of this ball is contained in the embedding ( $[5$, Theorem 8.1$]$; the proof in the nonorientable case is quite similar, since the only thing needed is the existence of a hierarchy [2]). Hence $\tilde{M}$ does not contain fake 3-cells, and since $\pi_{2}(\tilde{M})=0$ it follows that $\tilde{M}$ is irreducible. By the lemma, $\tilde{M}$ is a fiber bundle with fiber $F$, in particular $\tilde{M}$ is closed, which is absurd.

The first part of the proof gives us immediately:

Proposition. Let $M$ be a closed $P^{2}$-irreducible 3-manifold and sup- 
pose the center 3 of $\pi_{1}(M)$ is infinite. If $\pi_{1}(M)$ contains a subgroup $\mathfrak{F}$ then $F \times S^{1}$ is a covering of $M$.

Corollary (To Theorem 2). The groups

$$
\begin{aligned}
& \mid t_{1}, \cdots, t_{m}, g_{1}, \cdots, g_{n}, a_{1}, b_{1}, \cdots, a_{p}, b_{p}, h: \\
& \quad t_{i} h t_{i}^{-1}=h ; g_{i} h g_{i}^{-1}=h ; a_{i} h a_{i}^{-1}=h ; b_{i} h b_{i}^{-1}=h ; \\
& \quad \alpha_{i}^{\alpha_{i}} h^{\beta_{i}}=1,\left(\alpha_{i}, \beta_{i}\right)=1, t_{1} \cdots t_{m} g_{1} \cdots g_{n} \prod_{i=1}^{p}\left[a_{i}, b_{i}\right]=h^{b}, \quad b \in Z \mid
\end{aligned}
$$

do not contain a subgroup $\mathfrak{F}$.

These are fundamental groups of Seifert fiber spaces. In particular the groups of torus knots $\left|g, h: g^{\alpha} h^{\beta}=1\right|$ do not contain a subgroup $\mathfrak{F}$. Hence the complement of a torus knot does not contain closed incompressible surfaces other than Tori.

REMARK. The nonexistence of closed surfaces of genus $>1$ in irreducible orientable 3-manifolds $M$ with nonempty boundary for which $\pi_{1}(M)$ has nontrivial center follows immediately from Waldhausen's papers [6], [7]. In [6] Waldhausen proves that these manifolds are Seifert fiber spaces and in $[7, \S(10.3)]$ it is remarked that any incompressible surface in $M$ which is not boundary-parallel either consists of Seifert fibers (but does not contain singular fibers) or is a branched covering over the Seifert surface ("Zerlegungsfläche").

\section{REFERENCES}

1. D. B. A. Epstein, Projective planes in 3-manifolds, Proc. London Math. Soc. 3 (1961), 469-484.

2. W. H. Heil, On $P^{2}$-irreducible 3-manifolds, Bull. Amer. Math. Soc. 75 (1969), 772-775.

3. L. Neuwirth, Knot groups, Ann. of Math. Studies, no. 56, Princeton Univ. Press, Princeton, N. J., 1965.

4. J. Stallings, On fibering certain 3-manifolds, topology of 3-manifolds and related topics, Prentice-Hall, Englewood, N. J., 1962.

5. F. Waldhausen, On irreducible 3-manifolds which are sufficiently large, Ann. of Math. (2) 87 (1968), 56-88.

6. - Gruppen mit Zentrum und 3-dimensionale Mannigfaltigkeiten, Topology 6 (1967), 505-517.

7. - Eine Klasse von 3-dimensionalen Mannigfaltigkeiten. II, Invent. Math. 4 (1967), 87-117.

RICE UNIVERSITY 